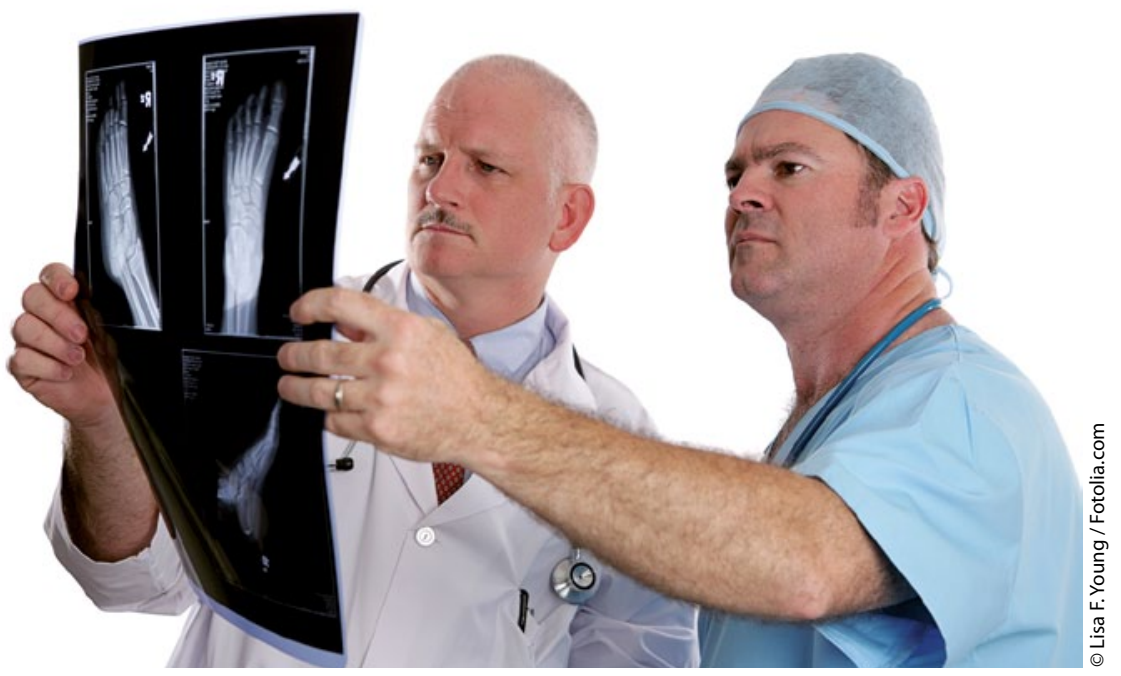

Werden honorar- und konsiliarärztliche Leistungen nicht direkt am Patienten erbracht, so können auch diese als steuerbefreite Heilbehandlung gelten.

\title{
Bundesfinanzhof entschärft das Umsatzsteuerrisiko
}

\author{
Gute Nachricht für Ärzte, die auch honorar- oder konsiliarärztliche \\ Leistungen erbringen: Die Leistungen können auch dann umsatzsteu- \\ erfrei sein, wenn sie nicht unmittelbar gegenüber einem Patienten \\ erbracht werden. Das hat der Bundesfinanzhof jetzt festgelegt.
}

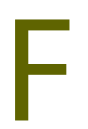

ür erhebliche Unruhe in Krankenhausverwaltungen und bei Ärzten sorgten jüngst Betriebsprüfungen, insbesondere in Bayern: Die Prüfer kamen vermehrt zu dem Ergebnis, dass honorarund konsiliarärztliche Tätigkeiten umsatzsteuerpflichtig sind. Viele Kliniken sind deshalb dazu übergegangen, Vertragsregelungen aufzunehmen, wonach Honorar- und Konsiliarärzte für die Anmeldung und Abführung der Umsatzsteuer selbst verantwortlich sind, um die eigene Haftung für eventuell nicht abgeführte Umsatzsteuer auszuschließen. Zum Ärgernis der Ärzte, die nun eine Verkürzung der Honorare um rund $16 \%$ des Nominalbetrages befürchteten. Das vom Krankenhaus gezahlte Entgelt ist als Bruttoentgelt anzusehen, bei dem die Umsatzsteuer herauszurechnen ist. Da als Honorar- oder Konsiliararzt tätige Mediziner aber häufig kaum umsatzsteuerpflichtige Ausgaben haben, führt die Umsatzsteuerpflicht beim Honorar zu einer unmittelbaren Einkommenseinbuße.
Nun hat der Bundesfinanzhof (BFH) entschieden: Infektionshygienische Leistungen eines Arztes, die dieser für andere Ärzte oder Kliniken erbringt, gelten immer dann als steuerfreie Heilbehandlungsleistungen, wenn die Klinik oder der andere Arzt damit seine Heilbehandlungsleistung ordnungsgemäß erbringen kann - unter Beachtung der für ihn nach dem Infektionsschutzgesetz bestehenden Verpflichtungen (Aktenzeichen V R 27/10). Gemäß $₫ 4$ Nr. 14 UStG sind Heilbehandlungsleistungen grundsätzlich umsatzsteuerfrei. Der Paragraf regelt auch die Umstände, die für eine Befreiung erfüllt sein müssen. Die Grundsätze, die der BFH in diesem Urteil aufstellt, weisen über den Infektionshygieniker allerdings hinaus. Letztlich entziehen sie der Rechtsauffassung der Finanzverwaltung, honorar- und konsiliarärztliche Tätigkeit sei umsatzsteuerpflichtig, den Boden.

Bisher hatte die Finanzverwaltung argumentiert, ärztliche Leistungen außerhalb von Kliniken und ähnlichen Einrich- tungen, also durch niedergelassene Ärzte, seien nur steuerbefreit, wenn diese im Rahmen eines persönlichen Vertrauensverhältnisses zwischen dem Patienten und dem Arzt in der Praxis, in der Wohnung des Patienten oder an einem anderen Ort erbracht werden. Da bei einem Honorarund Konsiliararzt keine besondere Vertrauensbeziehung zwischen Arzt und Patienten bestehe, der Patient vielmehr sozusagen zufällig von diesem Arzt behandelt werde, sei diese Tätigkeit nicht umsatzsteuerfrei.

Im Umsatzsteueranwendungserlass Ziffer 4.14.2 heißt es ausdrücklich: „Leistungen eines Arztes aus dem Betrieb eines Krankenhauses oder einer anderen Einrichtung im Sinne des $\$ 4$ Nr. 14 b UStG sind auch hinsichtlich der ärztlichen Leistungen nur dann befreit, wenn die in $\$ 4$ Nr. 14 b UStG bezeichneten Voraussetzungen erfüllt sind." Zwar gesteht die Finanzverwaltung zu, dass Heilbehandlungsleistungen eines selbstständigen Arztes, die in einem Krankenhaus erbracht werden (z. B. Belegarzt) sowie die selbstständigen ärztlichen Leistungen eines im Krankenhaus angestellten Arztes (z.B. in einer eigenen Praxis im Krankenhaus) nach $₫ 4$ Nr. 14 a UStG befreit sind. Nicht geregelt im Umsatzsteueranwendungserlass sind aber die Tätigkeiten des Honorar- und Konsiliararztes. Da diese Ärzte in Kliniken, anders als Ärzte mit eigener Praxis in der Klinik oder Belegärzte, keine direkten Behandlungsverträge mit Patienten abschließen, und auch nicht Leistungen für, sondern an die Klinik erbringen, sah die Finanzverwaltung eine Umsatzsteuerpflicht, da der Befreiungstatbestand nicht zähle, als gegeben an. Der BFH hat diese Regelungen nun ausdrücklich für falsch erklärt. Entgegen der Auffassung der Finanzverwaltung sei es nicht erforderlich, dass ärztliche Leistungen unmittelbar gegenüber einem Patienten erbracht würden, um als steuerbefreite Heilbehandlung zu gelten. Auch ein von der Finanzverwaltung gefordertes Vertrauensverhältnis zwischen Arzt und Patienten sei nicht zwingend für die Umsatzsteuerbefreiung.

\section{Dietmar Sedlaczek}

Steuerberater und Fachanwalt für Medizirecht, Kanzlei für Medizin- und Steuerrecht in Berlin, Partner des Steuerberaterverbunds Metax 